\title{
Circuit Realization of a 3D Multistability Chaotic System and Its Synchronization via Linear Resistor and Linear Capacitor in Parallel Coupling
}

\author{
Xikui Hu' ${ }^{1}$ and Ping Zhou (iD) ${ }^{1,2}$ \\ ${ }^{1}$ Center of System Theory and Its Applications, Chongqing University of Posts and Telecommunications, \\ Chongqing 400065, China \\ ${ }^{2}$ Key Laboratory of Network Control and Intelligent Instrument of Ministry of Education, \\ Chongqing University of Posts and Telecommunications, Chongqing 400065, China \\ Correspondence should be addressed to Ping Zhou; zhouping@cqupt.edu.cn
}

Received 5 December 2019; Revised 9 February 2020; Accepted 13 March 2020; Published 30 April 2020

Guest Editor: Viet-Thanh Pham

Copyright (C) 2020 Xikui Hu and Ping Zhou. This is an open access article distributed under the Creative Commons Attribution License, which permits unrestricted use, distribution, and reproduction in any medium, provided the original work is properly cited.

\begin{abstract}
In this paper, a 3D multistability chaotic system with two coexisting conditional symmetric attractors is studied by using a circuit block diagram and realized by using an electronic circuit. The simulation results show that two coexisting conditional symmetric attractors are emerged in this electronic circuit. Furthermore, synchronization of this 3D multistability chaotic system and its electronic circuit is studied. It shows that linear resistor and linear capacitor in parallel coupling can achieve synchronization in this chaotic electronic circuit. That is, the output voltage of chaotic electronic circuit is coupled via one linear resistor and one linear capacitor in parallel coupling. The simulation results verify that synchronization of the chaotic electronic circuit can be achieved.
\end{abstract}

\section{Introduction}

There are many nonlinear systems known to obtain coexistence of multiple attractors [1-10]. The coexistence of multiple attractors indicates that the attractor depends crucially on the initial condition (IC). These nonlinear systems are referred to as multistability systems. Multistability has been found in various systems, including Lorenz system [11], Rössler oscillators [12], neuronal oscillator [13], lasers [14], DC/DC converter [15], and permanent magnet synchronous motor [16]. Meanwhile, many multistability chaotic systems have been reported in recent years. Kengne et al. [17] reported a multistability chaotic system via van der Pol oscillator and suggested an appropriate electronic simulator. Peng and Min [18] proposed a novel multistability memristive chaotic circuit and applied it to image encryption. Chen et al. [19] introduced a multistability modified canonical Chua's circuit and obtained three sets of topologically different and disconnected attractors. Pham et al. [2] suggested a multistability chaotic system with no equilibrium.

On the other hand, synchronous behavior, which ensures that the states track the desired trajectory, has attracted much research attention for its potential applications especially in secure communication and image encryption [20]. Many chaotic electronic circuits reconstructed for chaotic attractors in nonlinear systems have been proposed. Therefore, synchronization of nonlinear chaotic systems can be converted to synchronization of chaotic electronic circuits. In recent years, linear capacitor coupling, linear resistor coupling, and linear inductor coupling have been used to achieve synchronization of two identical chaotic electronic circuits, in which many interesting results have been obtained. Liu et al. [21, 22] realized synchronization control for Chua's chaotic circuits and synchronization of neural circuits. Yao et al. [23] 


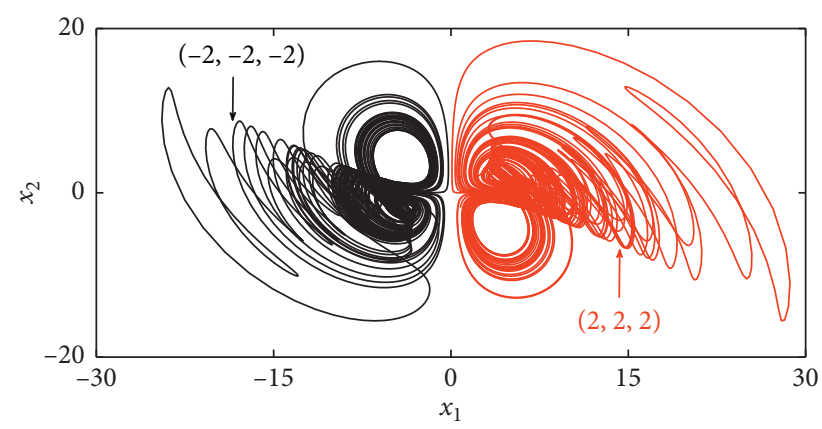

(a)

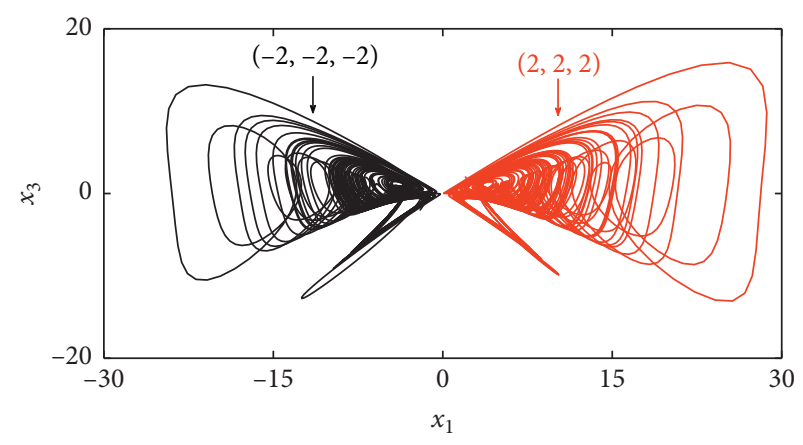

(b)

FIgURE 1: A symmetric pair of coexisting attractors in system (1) with $a=2.5$. IC $=(2,2,2)$ is red in the positive-x region and IC $=(-2,-2$, -2 ) is black in the negative-x region. (a) The $x_{1} x_{2}$ phase diagram and (b) the $x_{1} x_{3}$ phase diagram.

proposed a synchronization scheme for nonlinear circuits via induction coil coupling. Feng et al. [24] studied synchronization and electronic circuit application of a hidden hyperchaos system without equilibria. Singh and Roy [25] used adaptive contraction theory to research synchronization of a Lorenz hyperchaotic system and its circuit realization. He et al. [26] studied the dynamics and synchronization of conformable fractional-order hyperchaotic systems. Ma et al. [27] realized crack synchronization for chaotic circuits via field coupling. When the chaotic systems transform to nonlinear electronic circuits, direct linear variable coupling between chaotic systems can be implemented as a linear resistor coupling, and first derivative of state variable linear coupling can be implemented as a linear capacitive coupling or a linear inductor coupling. In fact, the synchronization of chaotic systems by resistor coupling is based on the consumption of Joule heat, and the synchronization of chaotic systems by capacitive coupling or inductor coupling is based on electric field energy exchange or magnetic field energy exchange.

Based on the 3D multistability chaotic system [1] reported by Zhou and Ke, in which there are two coexisting conditional symmetric chaotic attractors with different initial conditions, the chaos synchronization achieved by linear resistor and capacitor coupling is studied in this paper. First, the 3D multistability chaotic system [1] is studied by using a block diagram, and its electronic circuit is realized. The circuit simulation results are given. Second, the synchronization between two 3D multistability chaotic circuits is discussed, and we obtain that chaos synchronization can be achieved by using only one linear capacitor and one linear resistor in parallel coupling.

\section{A 3D Multistability Chaotic System with Two Coexisting Conditional Symmetric Attractors and Its Circuit Realization}

Based on the 3D Lü chaotic system [28], a multistability chaotic system with two coexisting conditional symmetric attractors has been reported by Zhou and Ke [1], which is shown as follows:

$$
\left\{\begin{array}{l}
\dot{x}_{1}=-x_{1}+0.5 x_{1} x_{3}+x_{2} x_{3}, \\
\dot{x}_{2}=a x_{2}-1.2 x_{1} x_{3}, \\
\dot{x}_{3}=x_{1} x_{2}-6 x_{3} .
\end{array}\right.
$$

When $0 \leq a \leq 4$, there are two coexisting conditional symmetric attractors in the positive- $x$ region and negative- $x$ region separately [1] with different initial conditions. For example, let $a=2.5$, the maximum Lyapunov exponent is 0.5758 [1]. The positive- $x$ region chaotic attractor with initial conditions $(2,2,2)$ and negative- $x$ region attractor with initial conditions $(-2,-2,-2)$ are shown in Figure 1, respectively.

Next, using the MATLAB Simulink module, circuit implementation of system (1) can be realized by block diagram in which all the blocks are standard basic operational circuits. Integrators marked as "Integrator" blocks are employed to obtain output voltage signal $v_{i}$ with input voltage signal $\dot{v}_{i}$. Without loss of generality, the value of resistor in each integrator is $R_{0}=100 \mathrm{k} \Omega$ and the value of capacitor is $C_{0}=10 \mathrm{nF}$ for dimensionless. The voltage signals $v_{i}$ are thus converted to dimensionless parameter $x_{i}$. All nonlinear terms $x_{i} x_{j}$ are obtained by using multipliers marked as "Product" blocks. For example, multiplier "Product $x_{1} x_{2}$ " is employed to produce output signal $x_{1} x_{2}$ with the input signals $x_{1}$ and $x_{2}$. All coefficients except " 1 " are implemented by using gain converters marked as "Gain" blocks. The gain converter is composed of an inverse proportional circuit with coefficient " $K=R_{f} / R_{K}$ " and an inverter is linked together. Similarly, the reference resistance is $R_{f}=100 \mathrm{k} \Omega$ for dimensionless. Therefore, the resistance with respect to the coefficient is $R_{K}=100 / K \mathrm{k} \Omega$. The output signal is $x_{\mathrm{o}}=K x_{i}$ with respect to the input signal $x_{i}$ in the "Gain" blocks, and $K$ is the gain coefficient marked inside the block. Adders marked as "Add" blocks are employed to realize addition and subtraction between the input signals. Finally, all the blocks can form three circuit loops as shown in Figure 2. Each loop corresponds to a dimensionless nonlinear equation in system (1).

In the implementation of system (1) with blocks, the properties of the chaotic system (1) can be studied by computer simulation experiment. The evolution of each signal $x_{i}(i=1,2,3)$ with respect to time $t$ can be demonstrated by "Scope" block connected with corresponding 


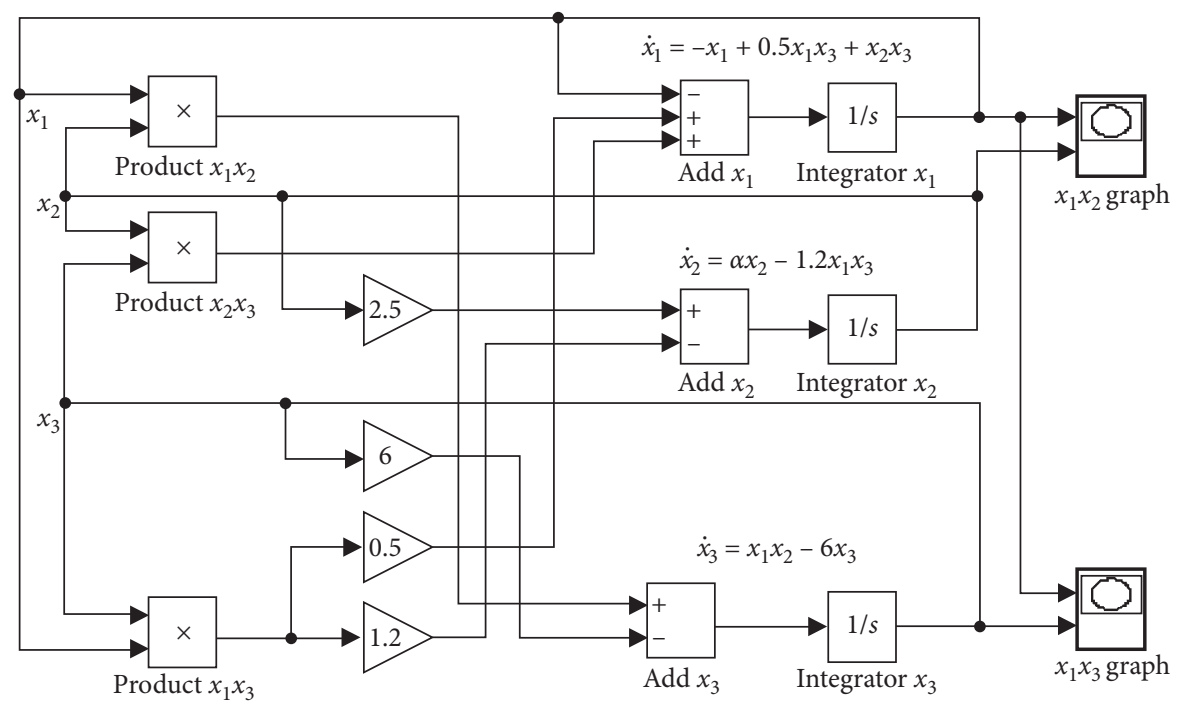

Figure 2: Implementation of system (1) realized by using the block diagram in the MATLAB simulink module.

signal. " $X Y$ Graph" blocks, which play the part of an oscilloscope with two vertical input signals at the same time, are employed in plotting the phase diagrams of two arbitrarily different signals $x_{i}$ and $x_{j}$. As shown in Figure 3, the phase diagrams of positive-x region attractors are observed with the " $x$ Initial $=\left[\begin{array}{lll}2 & 2 & 2\end{array}\right]$ " input into the MATLAB workspace, and the phase diagrams of negative- $x$ region attractors are observed with the " $x$ Initial $=\left[\begin{array}{lll}-2 & -2 & -2\end{array}\right]$." The results of circuit simulation by the MATLAB Simulink module fit well with that of nonlinear dynamic system (1).

The circuit simulation system based on the standard circuit described as blocks by the MATLAB Simulink module has the advantages of intuitionistic design, simple parameter setting, and easy debugging. In practical circuits, however, some blocks can be combined for economy. Multiple signals with parallel connection are adopted at the input terminal of the integrator in order to remove adders. The resistance of each branch in the input terminal is properly selected to remove gains. Finally, the electronic circuit can be obtained for practical application and the usage of electronic components can be greatly reduced. The electronic circuit of system (1) is shown in Figure 4. Without loss of generality, nonlinear terms $x_{i} x_{j}$ are obtained by using a multiplier with two signals $x_{i}$ and $x_{j}$ input at the same time and the minus of the signals is realized by using an inverter. $u$ represents the input terminal of the coupling signal which is suspended herein. It means that there is no coupling signal at this condition.

Nonlinear equations from the electronic circuit are derived as follows:

$$
\left\{\begin{array}{l}
C_{1} \frac{\mathrm{d} \dot{v}_{1}}{\mathrm{~d} \tau}=-\frac{v_{1}}{R_{11}}+\frac{v_{2} v_{3}}{R_{12}}+\frac{v_{1} v_{3}}{R_{13}}, \\
C_{2} \frac{\mathrm{d} \dot{v}_{2}}{\mathrm{~d} \tau}=\frac{v_{2}}{R_{21}}-\frac{v_{1} v_{3}}{R_{22}}, \\
C_{3} \frac{\mathrm{d} \dot{v}_{3}}{\mathrm{~d} \tau}=\frac{v_{1} v_{2}}{R_{31}}-\frac{v_{3}}{R_{32}} .
\end{array}\right.
$$

Note that we set $R_{0}=100 \mathrm{k} \Omega, C_{0}=10 \mathrm{nF}$, and the time scaling as $t_{0}=R_{0} C_{0}=10^{-3} \mathrm{~S}$. Let $x_{1}=v_{1}, x_{2}=v_{2}, x_{3}=v_{3}$, and $t=\tau / t_{0}$. A dimensionless dynamical system (3) mapped from the circuit equations can be approached as follows:

$$
\left\{\begin{array}{l}
\frac{C_{1}}{C_{0}} \frac{\mathrm{d} \dot{x}_{1}}{\mathrm{~d} t}=-\frac{R_{0}}{R_{11}} x_{1}+\frac{R_{0}}{R_{12}} x_{2} x_{3}+\frac{R_{0}}{R_{13}} x_{1} x_{3}, \\
\frac{C_{2}}{C_{0}} \frac{\mathrm{d} \dot{x}_{2}}{\mathrm{~d} t}=\frac{R_{0}}{R_{21}} x_{2}-\frac{R_{0}}{R_{22}} x_{1} x_{3}, \\
\frac{C_{3}}{C_{0}} \frac{\mathrm{d} \dot{x}_{3}}{\mathrm{~d} t}=\frac{R_{0}}{R_{31}} x_{1} x_{2}-\frac{R_{0}}{R_{32}} x_{3} .
\end{array}\right.
$$

It indicates that the resistance $R$ is scaled in $100 \mathrm{k} \Omega$, capacitance $C$ is scaled in $10 \mathrm{nF}$, and time $t$ is scaled in $1 \mathrm{~ms}$ when circuit equations are dimensionless.

\section{Synchronization of Multistability Chaotic System (1) by Using One Linear Capacitor and One Linear Resistor in Parallel Coupling}

In this section, synchronization of multistability chaotic system (1) is discussed. Let system (1) be the driving system. The response system with signals $y_{1}, y_{2}$, and $y_{3}$ is shown as follows:

$$
\left\{\begin{array}{l}
\dot{y}_{1}=-y_{1}+0.5 y_{1} y_{3}+y_{2} y_{3}, \\
\dot{y}_{2}=a y_{2}-1.2 y_{1} y_{3}, \\
\dot{y}_{3}=y_{1} y_{2}-6 y_{3} .
\end{array}\right.
$$

Analogous to system (1), the corresponding circuit schematic diagram of response system (2) can be obtained by the MATLAB Simulink model as shown in Figure 5.

In order to study the chaotic synchronization between driving system (1) and response system (4), the state variable $x_{2}$ of driving system (1) (i.e., the output voltage signal $x_{2}$ in 


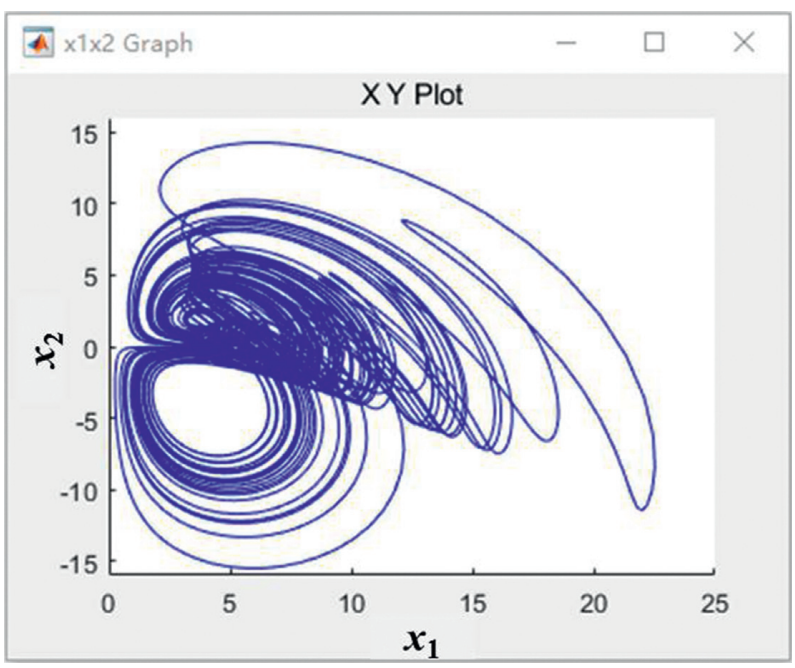

(a)

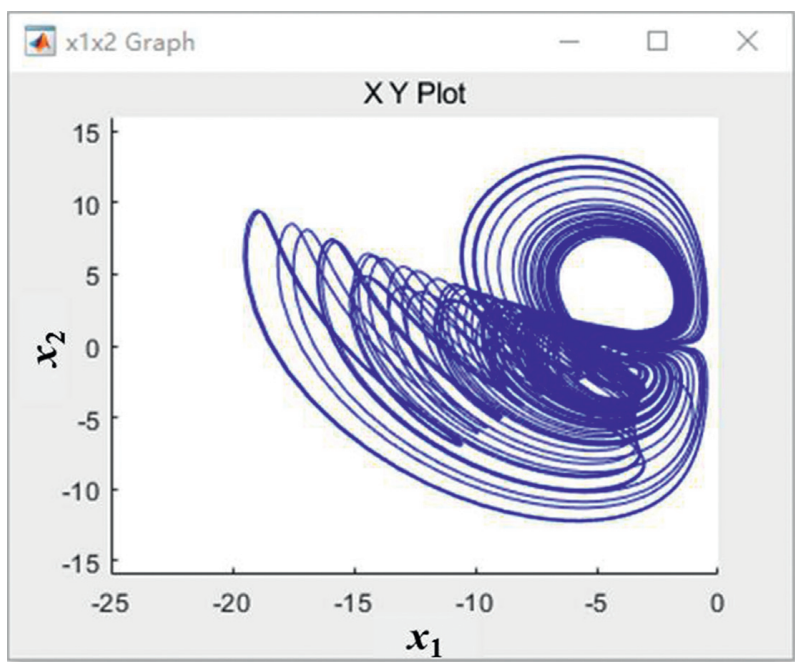

(c)

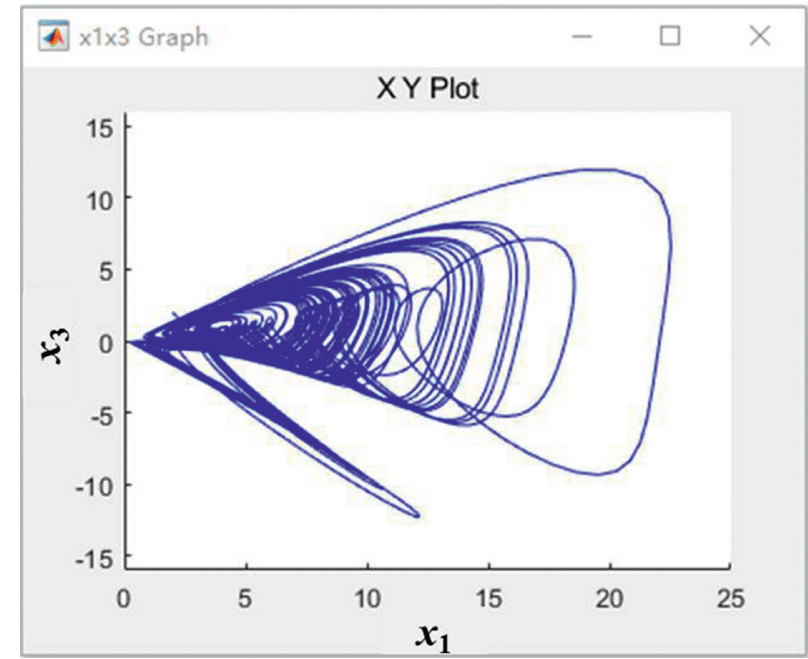

(b)

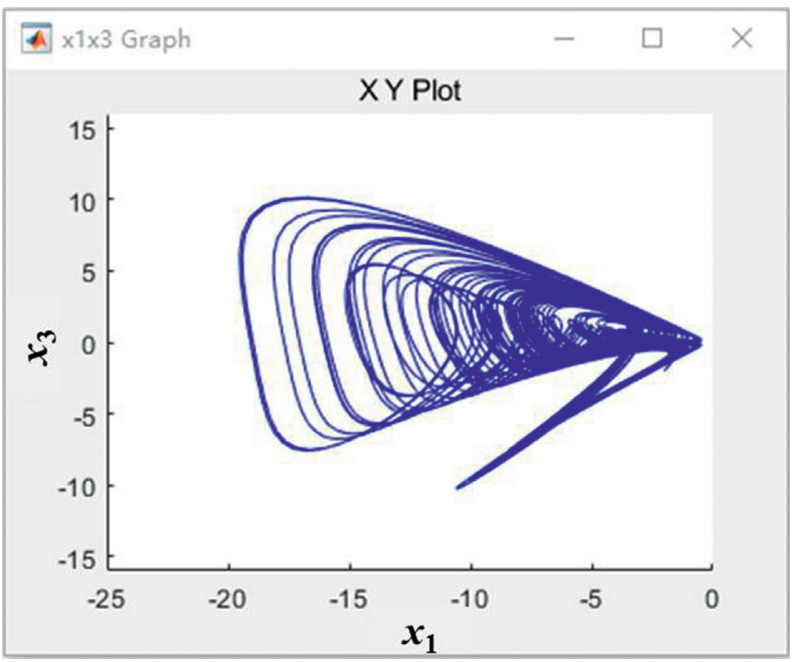

(d)

FIgURE 3: Formations of the phase diagrams that are plotted by "XY Graph" in the MATLAB Simulink module block diagram. (a) $x_{1} x_{2}$ phase diagram and (b) $x_{1} x_{3}$ phase diagram with $\mathrm{IC}=(2,2,2)$; (c) $x_{1} x_{2}$ phase diagram and (d) $x_{1} x_{3}$ phase diagram with $\mathrm{IC}=(-2,-2,-2)$.

Figure 2) and the state variable $y_{2}$ of response system (2) (i.e., the output voltage signal $y_{2}$ in Figure 5) are coupled in this paper. They are coupled via one linear resistor $R$ and one linear capacitor $C$ in parallel to form a new six-dimensional system in this paper. In order to obtain the dimensionless nonlinear equations of the coupled system, the unit of the coupling resistance $R$ is $100 \mathrm{k} \Omega$ and the unit of the coupling capacitance $C$ is $10 \mathrm{nF}$. The circuit implementation by blocks in the MATLAB Simulink module is shown in Figure 6. First, the subtraction circuit with $x_{2}$ and $y_{2}$ in the input terminal is used to obtain the output signal $x_{2}-y_{2}$. Second, the $x_{2}-y_{2}$ signal is divided into two branches. One branch is processed by a Gain block " $K_{R}$ " with the coefficient $K_{R}=100 \mathrm{k} \Omega / R$, which is equivalent to the resistive coupling. The corresponding output signal is $u_{R}=K_{R}\left(x_{2}-y_{2}\right)$. The other branch is processed by the combination of a Differentiator block and a Gain block " $K_{C}$ " with the coefficient $K_{C}=C / 10 \mathrm{nF}$, which is equivalent to the capacitive coupling. The corresponding output signal is $u_{C}=K_{C}\left(\dot{x}_{2}-\dot{y}_{2}\right)$.
After that, the two branches are combined together by an Add block to realize the parallel connection between the resistor and capacitor. The final output signal $u=K_{R}\left(x_{2}-y_{2}\right)+K_{C}\left(\dot{x}_{2}-\dot{y}_{2}\right)$, right now, is the coupling signal between driving system (1) and response system (4). The coupling strength is proportional to $K_{R}$ and $K_{C}$, which is inversely proportional to the value of coupling resistance $R$ and proportional to the value of coupling capacitance $C$, respectively. If the coupling resistance is close to zero, it is equivalent to a direct connection between $x_{2}$ and $y_{2}$. If the coupling resistance approaches infinity, it is equivalent to the coupling of a linear capacitor. If the coupling capacitance is close to zero, it is equivalent to the coupling of a linear resistor. At last, coupling signal $u$ is inverse feedback input to the adder of the second loop in the driving system and direct feedback input to the adder of the second loop in the response system, respectively. In this case, the second nonlinear equations of system (1) and system (4) are separately rewritten as 


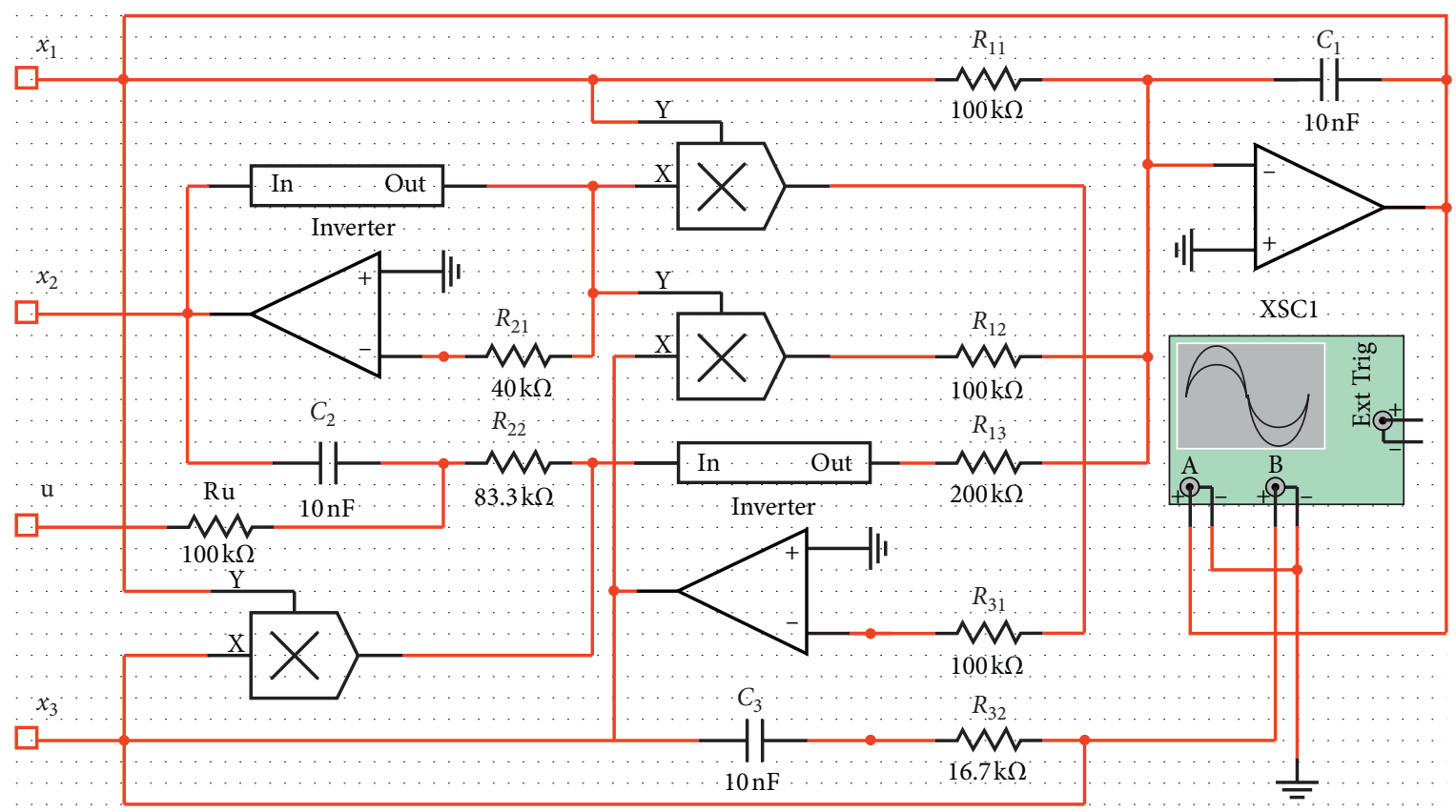

FIgURE 4: Schematic diagram of the electronic circuit realization of system (1).

$$
\left\{\begin{array}{l}
\dot{x}_{2}=a x_{2}-1.2 x_{1} x_{3}-u, \\
\dot{y}_{2}=a y_{2}-1.2 y_{1} y_{3}+u .
\end{array}\right.
$$

The electronic circuit described by using the block diagram in the MATLAB Simulink module can also be realized for practical applications as shown in Figure 7. Herein, the driving circuit and response circuit are represented by subcircuit blocks whose formations are shown in Figure 4. The signals $x_{2}$ and $y_{2}$ are connected to two input terminals of a subtraction circuit at the same time. The value of all the resistors is $100 \mathrm{k} \Omega$. The output signal of the subtraction circuit is $x_{2}-y_{2}$, which is then applied to both the resistor and capacitor concurrently. Without loss of generality, the unit of resistance $R$ is $100 \mathrm{k} \Omega$ and the unit of capacitance $C$ is $10 \mathrm{nF}$ in order to nondimensionalize the nonlinear equations of circuits. Besides, the coupling terminal in the driving system and response system connects with the feedback signals $u$ and $-u$, respectively.

When the coupled system is regarded as a new six-dimensional combined system, the dimensionless nonlinear state equations of coupled circuit (Figure 7) are described as

$$
\left\{\begin{array}{l}
\dot{x}_{1}=-x_{1}+0.5 x_{1} x_{3}+x_{2} x_{3}, \\
\dot{x}_{2}=a x_{2}-1.2 x_{1} x_{3}-\frac{\left(x_{2}-y_{2}\right)}{R}-C\left(\dot{x}_{2}-\dot{y}_{2}\right), \\
\dot{x}_{3}=x_{1} x_{2}-6 x_{3}, \\
\dot{y}_{1}=-y_{1}+0.5 y_{1} y_{3}+y_{2} y_{3}, \\
\dot{y}_{2}=a y_{2}-1.2 y_{1} y_{3}+\frac{\left(x_{2}-y_{2}\right)}{R} R+C\left(\dot{x}_{2}-\dot{y}_{2}\right), \\
\dot{y}_{3}=y_{1} y_{2}-6 y_{3} .
\end{array}\right.
$$

Herein, the parameter $a=2.5$, the unit of coupled resistance $R$ is $100 \mathrm{k} \Omega$, and the unit of coupled capacitance $C$ is $10 \mathrm{nF}$ for dimensionless as mentioned above. The nonlinear system (6) can be rewritten as nonlinear system:

$$
\left\{\begin{array}{l}
\dot{x}_{1}=-x_{1}+0.5 x_{1} x_{3}+x_{2} x_{3}, \\
\dot{x}_{2}=\frac{\left((1+C) f_{x}+C f_{y}\right)}{(1+2 C)}, \\
\dot{x}_{3}=x_{1} x_{2}-6 x_{3}, \\
\dot{y}_{1}=-y_{1}+0.5 y_{1} y_{3}+y_{2} y_{3}, \\
\dot{y}_{2}=\frac{\left(C f_{x}+(1+C) f_{y}\right)}{(1+2 C)}, \\
\dot{y}_{3}=y_{1} y_{2}-6 y_{3} .
\end{array}\right.
$$

Herein, $f_{x}$ and $f_{y}$ are introduced to simplify the form of the nonlinear equations of system (6). Their expressions are as follows:

$$
\left\{\begin{array}{l}
f_{x}=a x_{2}-1.2 x_{1} x_{3}-\frac{\left(x_{2}-y_{2}\right)}{R}, \\
f_{y}=a y_{2}-1.2 y_{1} y_{3}+\frac{\left(x_{2}-y_{2}\right)}{R} .
\end{array}\right.
$$

In order to study the chaotic evolution of system (7), especially the synchronization between the driving system and response system, the difference $e$ should be employed as follows: 


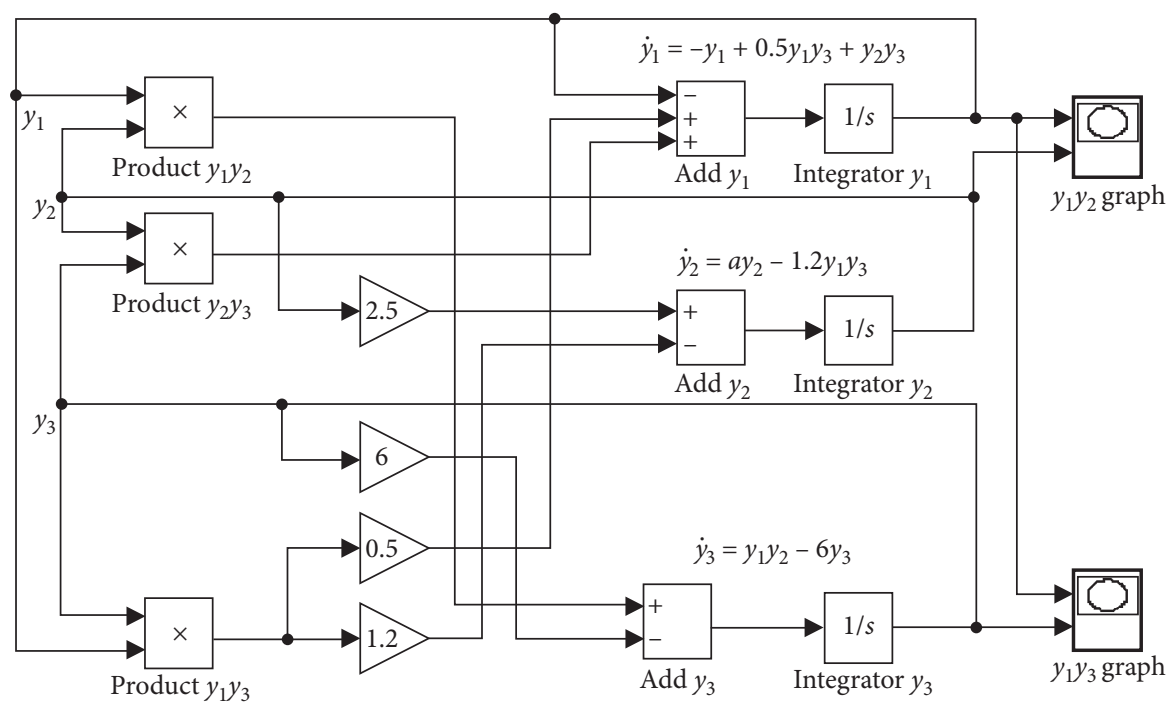

Figure 5: Implementation of response system (2) realized by block diagram in the MATLAB Simulink module.

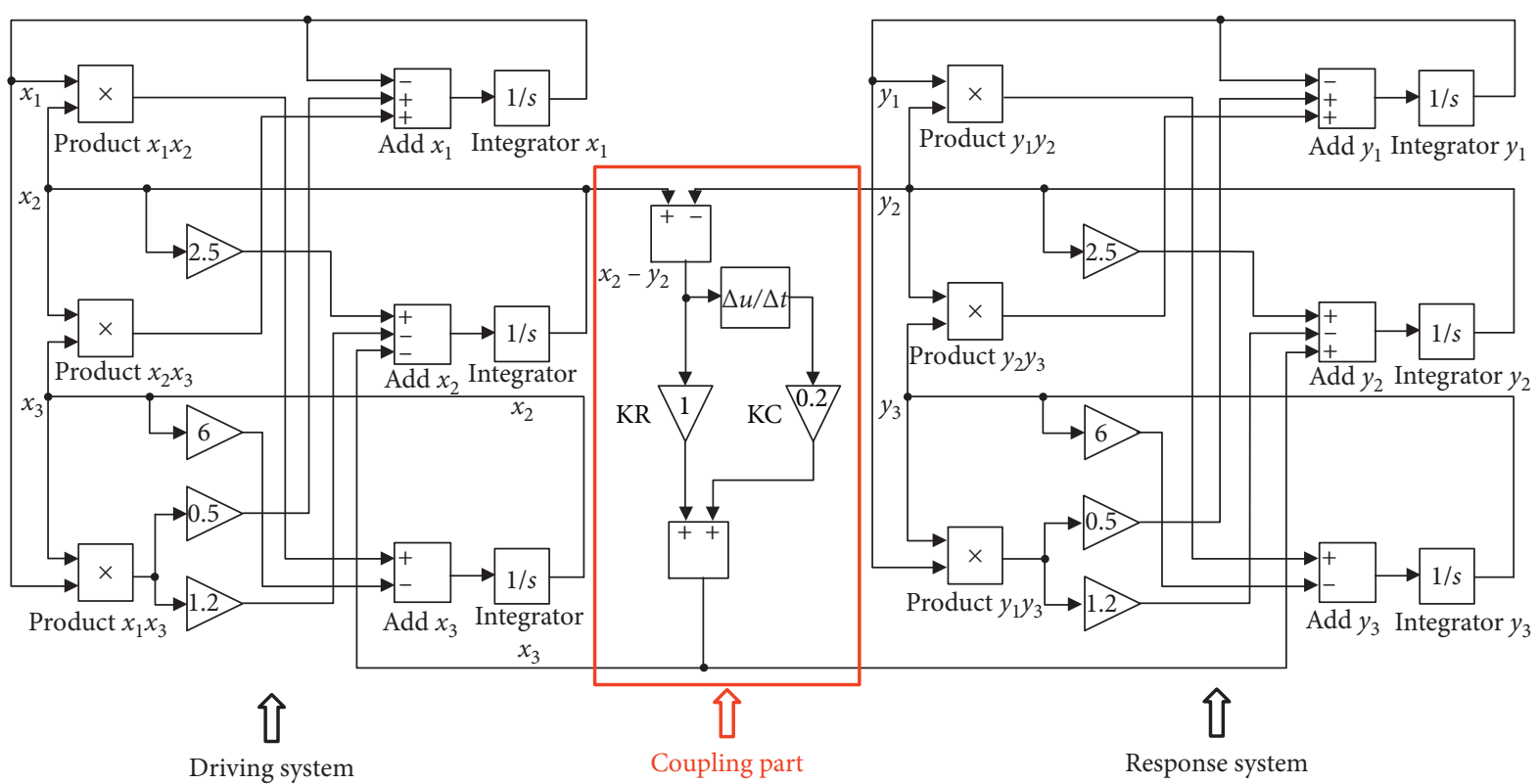

Figure 6: Implementation of the coupled system realized by block diagram in the MATLAB Simulink module.

$$
\begin{aligned}
& e_{1}=x_{1}-y_{1} \\
& e_{2}=x_{2}-y_{2} \\
& e_{3}=x_{3}-y_{3} .
\end{aligned}
$$

The corresponding error functions with respect to difference $e$ and driving signal $x$ are described as error system:

$$
\left\{\begin{array}{l}
\dot{e}_{1}=\left(-1+0.5 x_{3}\right) e_{1}+x_{3} e_{2}+\left(0.5 x_{1}+x_{2}\right) e_{3}-0.5 e_{1} e_{3}-e_{2} e_{3}, \\
\dot{e}_{2}=\frac{\left(-1.2 x_{3} e_{1}+(a-2 / R) e_{2}-1.2 x_{1} e_{3}+1.2 e_{1} e_{3}\right)}{(1+2 C)} \\
\dot{e}_{3}=x_{2} e_{1}+x_{1} e_{2}-6 e_{3}-e_{1} e_{2} .
\end{array}\right.
$$

It is obvious that $e=0$ is the equilibrium point of error system (10). If equilibrium point $e=0$ is asymptotic stability, then chaotic synchronization between driving system (1) and response system (4) can be achieved. It indicates that there exists a synchronized state $x=y$ for driving system (1) and response system (4). In general, the synchronization can be checked numerically by conditional Lyapunov exponents (CLEs). This is that synchronization occurs only if all CLEs of error system (10) are negative.

Therefore, the CLEs of system (10) are studied by MATLAB based on the QR decomposition method to analyse the synchronization with respect to the variable parameters $R$ and $C$. The Jacobi matrix of the error system (10) is 


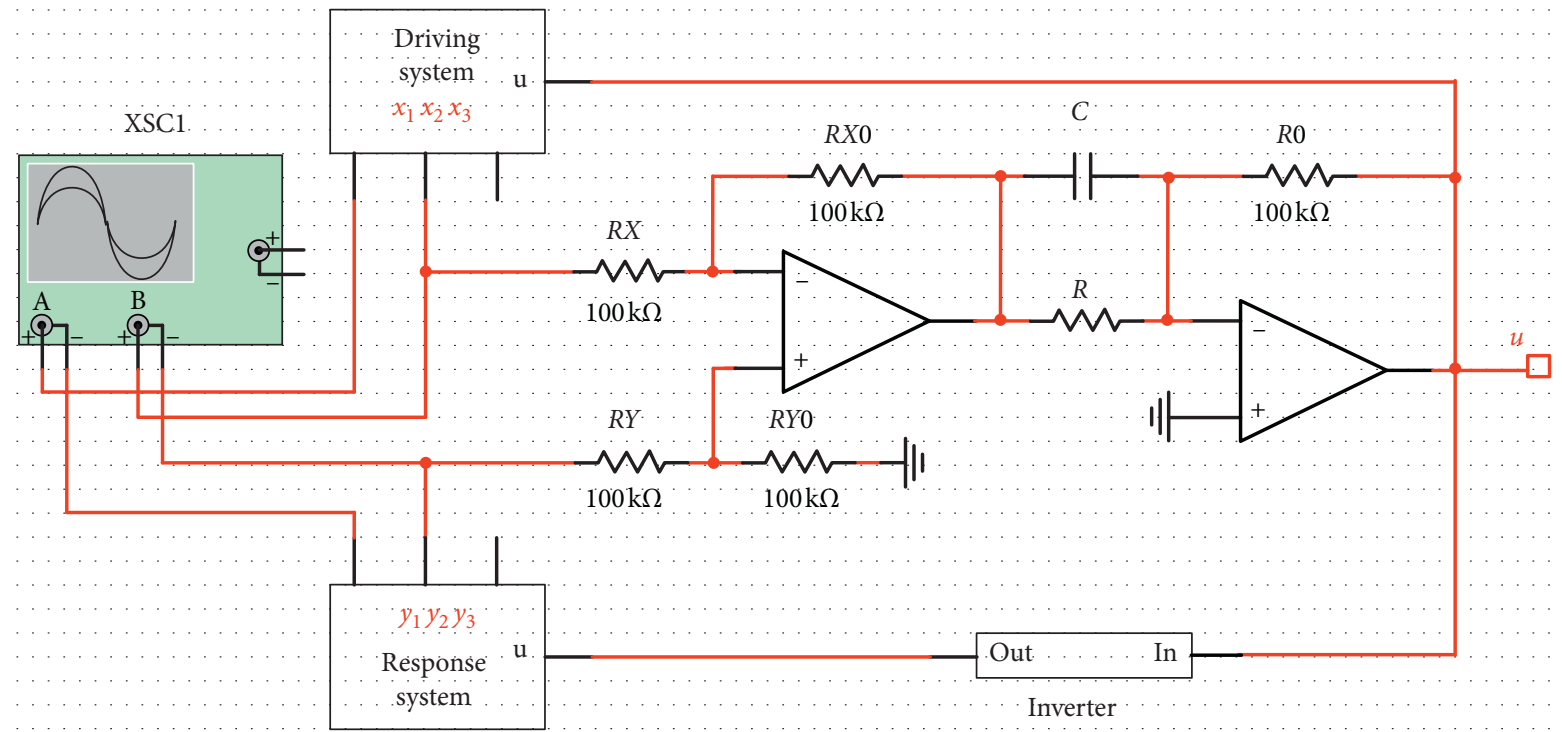

FIGURE 7: Schematic diagram of the electronic circuit realization of the coupled system.

$$
J=\left[\begin{array}{ccc}
-1+0.5\left(x_{3}-e_{3}\right) & x_{3}-e_{3} & 0.5\left(x_{1}-e_{1}\right)+\left(x_{2}-e_{2}\right) \\
\frac{-1.2\left(x_{3}-e_{3}\right)}{1+2 C} & \frac{a-2 / R}{1+2 C} & \frac{-1.2\left(x_{1}-e_{1}\right)}{1+2 C} \\
x_{2}-e_{2} & x_{1}-e_{1} & -6
\end{array}\right]
$$

All the CLEs have been calculated by MATLAB numerical simulation with initial driving signals $x_{0}=(2,2,2)$ and initial difference $e_{0}=(-1,-1,-1)$. The maximum CLEs distribution with respect to $R$ and $C$ is shown in Figure 8. It can be pointed out that the maximum CLEs are negative in the blue area and the maximum CLEs are positive in the yellow area. Therefore, the synchronization is realizable when the values of the coupling resistor and coupling capacitor are located in the blue area, while it is irrealizable when their values are in the yellow area. With the increase of capacitance, the range of resistance synchronization achieved is decreasing. When the capacitance $C>10 \mathrm{nF}$, synchronization scarcely exists. Synchronization also disappears when the resistance $R$ is much larger, e.g., $R>160 \mathrm{k} \Omega$.

Take $R=1.0$ and $C=0.2$; namely, the value of coupling resistance is $100 \mathrm{k} \Omega$ and coupling capacitance is $2 \mathrm{nF}$ as an example. In this case, the corresponding coefficients are $K_{R}=1$ and $K_{C}=0.2$. All the three CLEs of error system (10) are negative as $\lambda_{1}=-0.19, \lambda_{2}=-1.05$, and $\lambda_{3}=-5.50$. The equilibrium point $e=0$ in error system (10) is asymptotic stability. Therefore, synchronization exists in the coupled system (6). It is proved by circuit simulation of the MATLAB Simulink module as shown in Figure 9. Herein, the coefficient in the Gain block " $K_{\mathrm{R}}$ " is 1 and the coefficient in the Gain block " $K_{\mathrm{C}}$ " is 0.2 . Subtraction block is employed to obtain the difference signal. The input terminals are connected with $x_{i}$ and $y_{i}$, respectively; thus, the output signal of

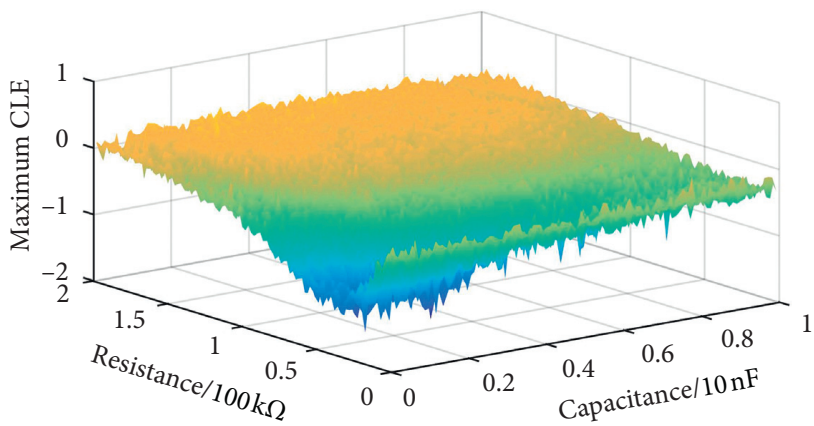

Figure 8: Maximum CLEs of error system (10) with resistive and capacitive coupling by parallel connection.

the Subtraction block is $e_{i}=x_{i}-y_{i}$. Scope block connected with the output terminal of the Subtraction block is used to plot the variation of difference signal $e_{i}$ with respect to $t$. As shown in Figure 9, it is obvious that all the three difference signals $e_{i}(t)$ gradually approach zero over some time with " $x$ Initial $=\left[\begin{array}{lll}2 & 2 & 2\end{array}\right]$ " and " $y$ Initial $=\left[\begin{array}{lll}3 & 3 & 3\end{array}\right]$ " are input into the MATLAB workspace. Therefore, it is confirmed that system (6) can achieve complete synchronization.

Generally, the synchronization performance varies with coupling parameters. As shown in Figure 8, the maximum CLEs of system (8) increase with respect to $C$ approximately when $R$ is determined. It means that the synchronization process slows down as $C$ increases. Absolute error $\operatorname{err}(t)$ is employed to estimate the synchronization process:

$$
\operatorname{err}(t)=\sqrt{e_{1}^{2}+e_{2}^{2}+e_{3}^{2}}
$$

As shown in Figure 10, absolute errors of the synchronization process with different $C$ values and $R=1.0$ are calculated. It can be verified that the larger the capacitance is, the longer the time will be taken to achieve synchronization. 

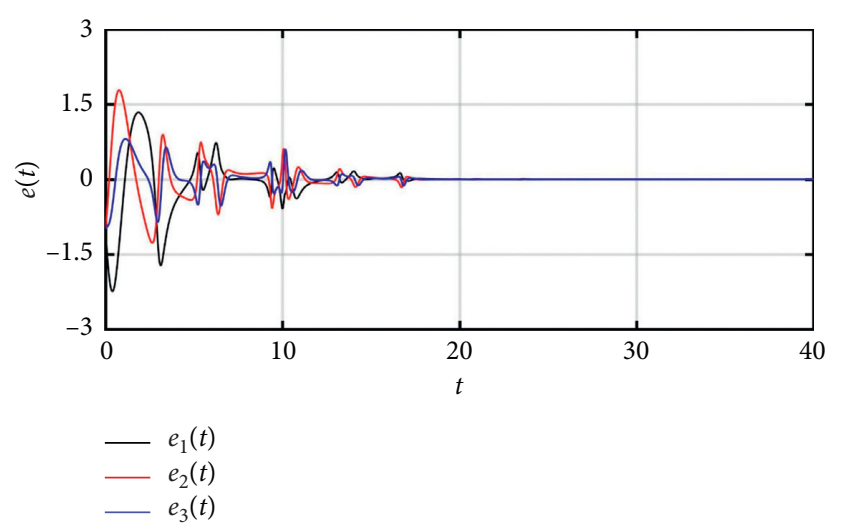

FIgURE 9: Variation of the difference $e$ with $R=1.0$ and $C=0.2$.

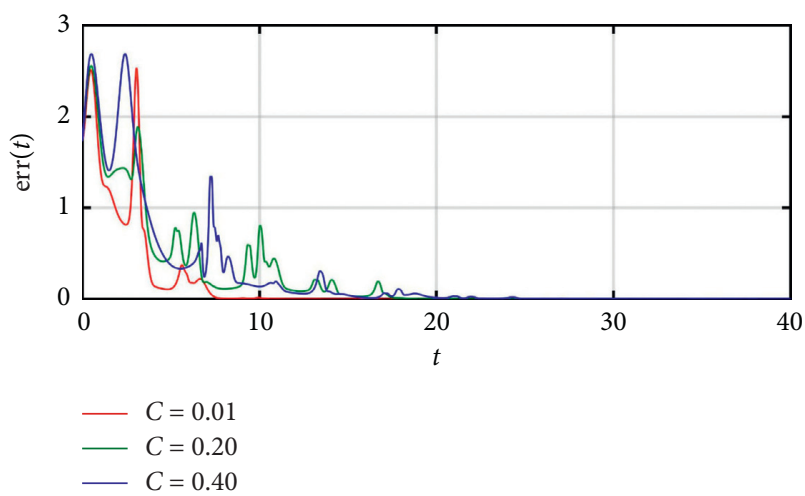

FIgUre 10: Variation of the absolute error $\operatorname{err}(t)$ with different $C$ values and $R=1.0$.

In addition, the only resistive coupling is also studied. In this condition, the coupling capacitance $C=0$ and the nonlinear system (6) are changed as follows:

$$
\left\{\begin{array}{l}
\dot{x}_{1}=-x_{1}+0.5 x_{1} x_{3}+x_{2} x_{3}, \\
\dot{x}_{2}=a x_{2}-1.2 x_{1} x_{3}-\frac{\left(x_{2}-y_{2}\right)}{R} \\
\dot{x}_{3}=x_{1} x_{2}-6 x_{3} \\
\dot{y}_{1}=-y_{1}+0.5 y_{1} y_{3}+y_{2} y_{3} \\
\dot{y}_{2}=a y_{2}-1.2 y_{1} y_{3}+\frac{\left(x_{2}-y_{2}\right)}{R} \\
\dot{y}_{3}=y_{1} y_{2}-6 y_{3} .
\end{array}\right.
$$

The corresponding error system is

$$
\left\{\begin{array}{l}
\dot{e}_{1}=\left(-1+0.5 x_{3}\right) e_{1}+x_{3} e_{2}+\left(0.5 x_{1}+x_{2}\right) e_{3}-0.5 e_{1} e_{3}-e_{2} e_{3}, \\
\dot{e}_{2}=-1.2 x_{3} e_{1}+\left(a-\frac{2}{R}\right) e_{2}-1.2 x_{1} e_{3}+1.2 e_{1} e_{3}, \\
\dot{e}_{3}=x_{2} e_{1}+x_{1} e_{2}-6 e_{3}-e_{1} e_{2} .
\end{array}\right.
$$

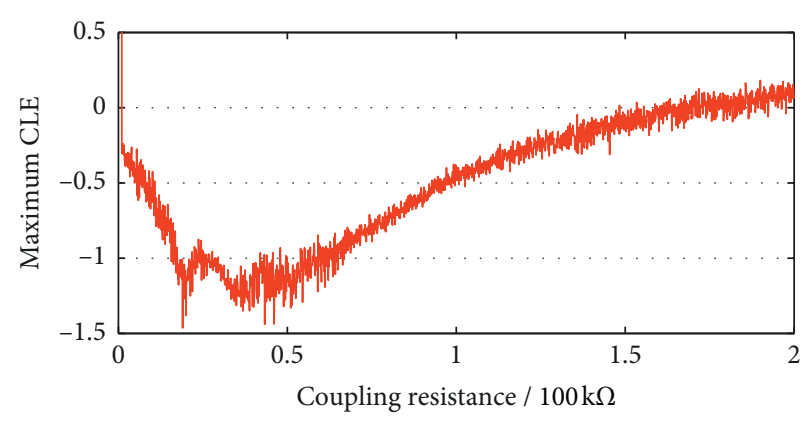

Figure 11: Maximum CLEs of the error system (14) with only resistive coupling.

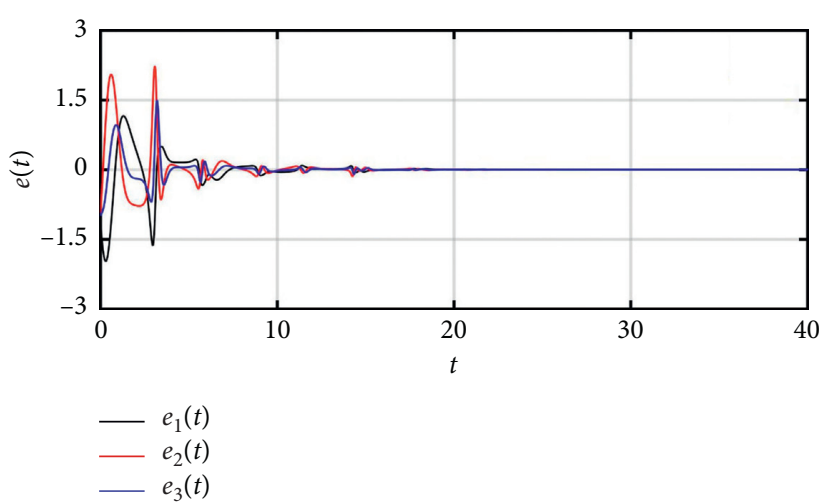

Figure 12: Variation of the difference $e$ with $R=1.0$.

The Jacobi matrix is

$J=\left[\begin{array}{ccc}-1+0.5\left(x_{3}-e_{3}\right) & x_{3}-e_{3} & 0.5\left(x_{1}-e_{1}\right)+\left(x_{2}-e_{2}\right) \\ -1.2\left(x_{3}-e_{3}\right) & a-\frac{2}{R} & -1.2\left(x_{1}-e_{1}\right) \\ x_{2}-e_{2} & x_{1}-e_{1} & -6\end{array}\right]$.

All the CLEs have been calculated by MATLAB numerical simulation with initial driving signals $x_{0}=(2,2,2)$ and initial difference $e_{0}=(-1,-1,-1)$ similarly. The maximum CLEs distribution with respect to $R$ is shown in Figure 11. It can be obtained that the maximum CLEs are negative when $R<1.6$. It means that synchronization is achieved when the value of coupling resistance is less than $160 \mathrm{k} \Omega$ for only resistance coupling condition.

Take $R=1.0$; namely, the value of coupling resistance is $100 \mathrm{k} \Omega$ as an example. In this case, the corresponding coefficients of Gain block " $K_{\mathrm{R}}$ " is $K_{R}=1$, while the corresponding coefficients of Gain block " $K_{C}$ " is $K_{C}=0$ which means the branch of capacitive coupling can even be removed. All the three CLEs of error system (14) are negative as $\lambda_{1}=-0.49$, $\lambda_{2}=-0.71$, and $\lambda_{3}=-5.30$. It indicates that synchronization exists in coupled system (13). As shown in Figure 12, it is obvious that all the three difference signals $e_{i}(t)$ gradually approach zero over some time with " $x$ Initial $=\left[\begin{array}{lll}2 & 2 & 2\end{array}\right]$ " and $" y$ Initial $=\left[\begin{array}{lll}3 & 3 & 3\end{array}\right] "$ input into the MATLAB workspace. 


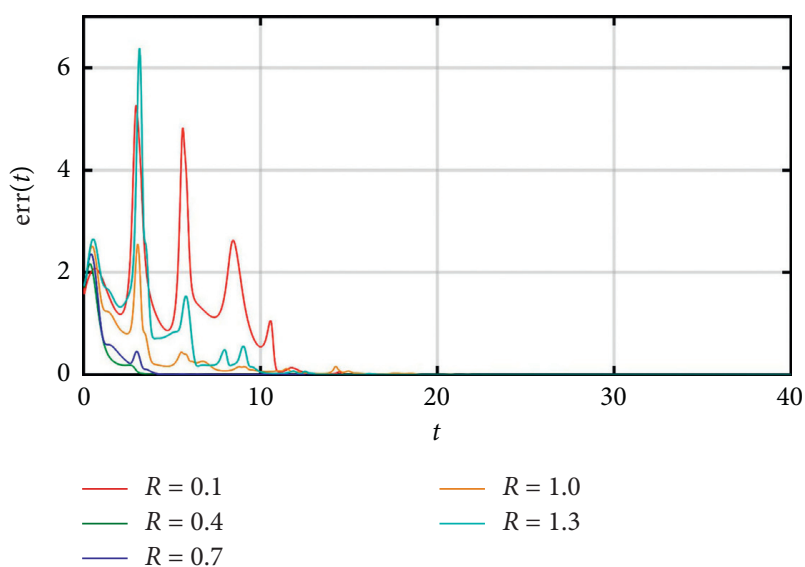

FIGURE 13: Variation of the absolute error $\operatorname{err}(t)$ with different $R$.

Therefore, it is confirmed that system (6) can achieve complete synchronization with suitable values (Figure 12).

Furthermore, the synchronization performance varies with $R$. As shown in Figure 11, the maximum CLEs of system (14) decrease at first and then increase with $R$ increasing approximately. Therefore, the synchronization process speeds up at first and then slows down as $R$ increases. As shown in Figure 13, absolute errors of the synchronization process with different $R$ values are calculated. It can be found out that the time taken to achieve synchronization reduces first and then increases when $R$ increases.

\section{Conclusions}

Based on a 3D multistability chaotic system [1] reported by Zhou and $\mathrm{Ke}$, an electronic circuit is proposed in this paper. The circuit simulation results show that there are two coexisting conditional symmetric chaotic attractors for different initial conditions, which are consistent with the findings in the reference [1]. Meanwhile, the chaotic synchronization between two 3D multistability chaotic systems with only one linear resistor and one linear capacitor in parallel coupling is discussed. The maximum condition Lyapunov exponents (CLEs) of the coupled system are studied. The negative maximum CLEs indicate that chaotic synchronization can be achieved with a capacitor and resistor in parallel coupling in the appropriate range. Furthermore, an electronic circuit is given to verify the synchronization scheme. Circuit simulation results confirm that the chaos synchronization for the $3 \mathrm{D}$ multistability chaotic system can be realized. Our work provides a method to realize the electronic circuit of the 3D multistability chaotic system and its synchronization, which has application prospect in secret communications and adaptive control. Future work can include the analysis of the synchronization between positive- $x$ region attractors and negative- $x$ region attractors.

\section{Data Availability}

The data used in our manuscript are obtained by MATLAB program and MATLAB Simulink module (MSM) and are available from the corresponding author upon request.

\section{Conflicts of Interest}

The authors declare that there are no conflicts of interest regarding the publication of this paper.

\section{References}

[1] P. Zhou and M. Ke, "A new 3D autonomous continuous system with two isolated chaotic attractors and its topological horseshoes," Complexity, vol. 2017, Article ID 4037682, 7 pages, 2017.

[2] V.-T. Pham, C. Volos, S. Jafari, and T. Kapitaniak, "Coexistence of hidden chaotic attractors in a novel no-equilibrium system," Nonlinear Dynamics, vol. 87, no. 3, pp. 2001-2010, 2017.

[3] S. Cang, Y. Li, R. Zhang, and Z. Wang, "Hidden and selfexcited coexisting attractors in a Lorenz-like system with two equilibrium points," Nonlinear Dynamics, vol. 95, no. 1, pp. 381-390, 2019.

[4] Q. Lai and S. Chen, "Coexisting attractors generated from a new 4D smooth chaotic system," International Journal of Control, Automation and Systems, vol. 14, no. 4, pp. 11241131, 2016.

[5] N. Stankevich and E. Volkov, "Multistability in a three-dimensional oscillator: tori, resonant cycles and chaos," Nonlinear Dynamics, vol. 94, no. 4, pp. 2455-2467, 2018.

[6] J. Kengne, Z. T. Njitacke, and H. B. Fotsin, "Dynamical analysis of a simple autonomous jerk system with multiple attractors," Nonlinear Dynamics, vol. 83, no. 1-2, pp. 751-765, 2016.

[7] C. B. Li, T. A. Lu, G. R. Chen, and H. Y. Xing, "Doubling the coexisting attractors," Chaos: An Interdisciplinary Journal of Nonlinear Science, vol. 29, no. 5, Article ID 051102, 2019.

[8] İ. Pehlivan, E. Kurt, Q. Lai, A. Basaran, and M. C. Kutlu, "A multiscroll chaotic attractor and its electronic circuit implementation," Chaos Theory and Applications, vol. 1, no. 1, pp. 29-37, 2019.

[9] C. K. Volos, A. Akgul, V. T. Pham, and M. S. Baptista, "Antimonotonicity, crisis and multiple attractors in a simple memristive circuit," Journal of Circuits, Systems and Computers, vol. 27, no. 2, Article ID 1850026, 2018.

[10] K. Rajagopal, J. P. Singh, B. K. Roy, and A. Karthikeyan, "Dissipative and conservative chaotic nature of a new quasiperiodically forced oscillator with megastability," Chinese Journal of Physics, vol. 58, pp. 263-272, 2019.

[11] C. B. Li and J. C. Sprott, "Multistability in the Lorenz system: a broken butterfly," International Journal of Bifurcation and Chaos, vol. 24, no. 10, Article ID 1450131, 2014.

[12] P. Jaros, P. Perlikowski, and T. Kapitaniak, "Synchronization and multistability in the ring of modified Rössler oscillators," The European Physical Journal Special Topics, vol. 224, no. 8, pp. 1541-1552, 2015.

[13] E. B. M. Ngouonkadi, H. B. Fotsin, P. L. Fotso, V. K. Tamba, and H. A. Cerdeira, "Bifurcations and multistability in the extended Hindmarsh-Rose neuronal oscillator," Chaos, Solitons \& Fractals, vol. 85, pp. 151-163, 2016.

[14] R. Meucci, E. Allaria, F. Salvadori, and F. T. Arecchi, "Attractor selection in chaotic dynamics," Physical Review Letters, vol. 95, no. 18, Article ID 184101, 2005.

[15] Z. T. Zhusubaliyev and E. Mosekilde, "Multistability and hidden attractors in a multilevel DC/DC converter," Mathematics and Computers in Simulation, vol. 109, pp. 32-45, 2015. 
[16] J. P. Singh, B. K. Roy, and N. V. Kuznetsov, "Multistability and hidden attractors in the dynamics of permanent magnet synchronous motor," International Journal of Bifurcation and Chaos, vol. 29, no. 4, Article ID 1950056, 2019.

[17] J. Kengne, J. C. Chedjou, M. Kom, K. Kyamakya, and V. K. Tamba, "Regular oscillations, chaos, and multistability in a system of two coupled van der Pol oscillators: numerical and experimental studies," Nonlinear Dynamics, vol. 76, no. 2, pp. 1119-1132, 2014.

[18] G. Peng and F. Min, "Multistability analysis, circuit implementations and application in image encryption of a novel memristive chaotic circuit," Nonlinear Dynamics, vol. 90, no. 3, pp. 1607-1625, 2017.

[19] M. Chen, Q. Xu, Y. Lin, and B. Bao, "Multistability induced by two symmetric stable node-foci in modified canonical Chua's circuit," Nonlinear Dynamics, vol. 87, no. 2, pp. 789-802, 2017.

[20] Q. Xu, K. Sun, C. Cao, and C. Zhu, "A fast image encryption algorithm based on compressive sensing and hyperchaotic map," Optics and Lasers in Engineering, vol. 121, pp. 203-214, 2019.

[21] Z. Liu, J. Ma, G. Zhang, and Y. Zhang, "Synchronization control between two Chua's circuits via capacitive coupling," Applied Mathematics and Computation, vol. 360, pp. 94-106, 2019.

[22] Z. Liu, C. Wang, W. Jin, and J. Ma, "Capacitor coupling induces synchronization between neural circuits," Nonlinear Dynamics, vol. 97, no. 4, pp. 2661-2673, 2019.

[23] Z. Yao, J. Ma, Y. Yao, and C. Wang, "Synchronization realization between two nonlinear circuits via an induction coil coupling," Nonlinear Dynamics, vol. 96, no. 1, pp. 205-217, 2019.

[24] Y. Feng, Z. Wei, U. E. Kocamaz, A. Akgül, and I. Moroz, "Synchronization and electronic circuit application of hidden hyperchaos in a four-dimensional self-exciting homopolar disc dynamo without equilibria," Complexity, vol. 2017, Article ID 7101927, 11 pages, 2017.

[25] J. P. Singh and B. K. Roy, "Hidden attractors in a new complex generalised Lorenz hyperchaotic system, its synchronisation using adaptive contraction theory, circuit validation and application," Nonlinear Dynamics, vol. 92, no. 2, pp. 373-394, 2018.

[26] S. He, K. Sun, and H. Wang, "Dynamics and synchronization of conformable fractional-order hyperchaotic systems using the Homotopy analysis method," Communications in Nonlinear Science and Numerical Simulation, vol. 73, pp. 146-164, 2019.

[27] J. Ma, F. Wu, A. Alsaedi, and J. Tang, "Crack synchronization of chaotic circuits under field coupling," Nonlinear Dynamics, vol. 93, no. 4, pp. 2057-2069, 2018.

[28] J. Lü and G. Chen, "A new chaotic attractor coined," International Journal of Bifurcation and Chaos, vol. 12, no. 3, pp. 659-661, 2002. 